Document downloaded from:

http://hdl.handle.net/10251/50495

This paper must be cited as:

Ballester-Bolinches, A.; Beidleman, JC.; Esteban Romero, R.; Ragland, MF. (2014). On a class of supersoluble groups. Bulletin of the Australian Mathematical Society. 90(2):220226. doi:10.1017/S0004972714000306.

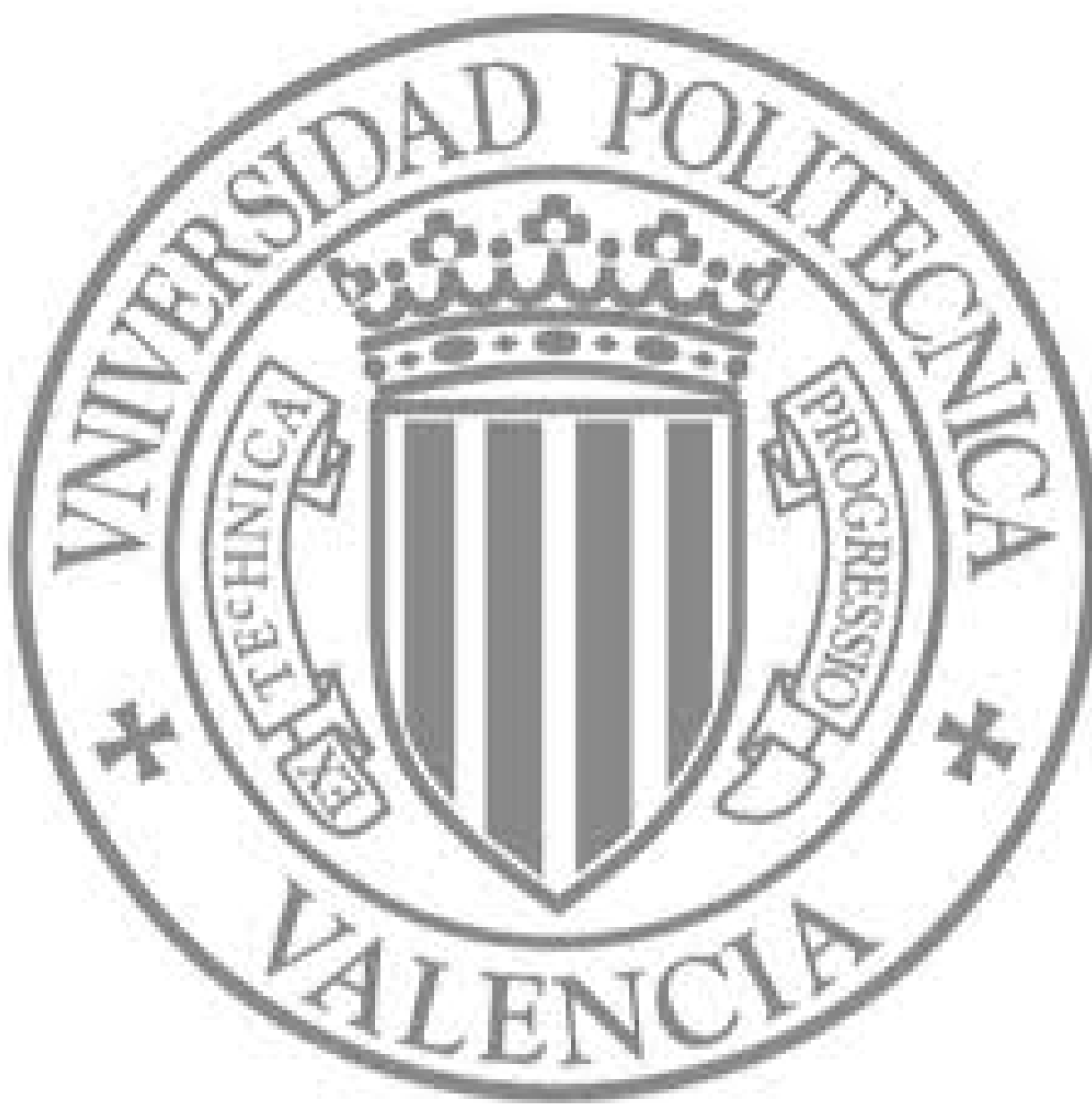

The final publication is available at

http://dx.doi.org/10.1017/S0004972714000306

Copyright Cambridge University Press (CUP): STM Journals - No Cambridge Open 


\title{
On a class of supersoluble groups
}

\author{
A. Ballester-Bolinches* \\ J. C. Beidleman ${ }^{\dagger}$ \\ R. Esteban-Romero \\ M. F. Ragland ${ }^{\S}$
}

\begin{abstract}
A subgroup $H$ of a finite group $G$ is said to be $S$-semipermutable in $G$ if $H$ permutes with every Sylow $q$-subgroup of $G$ for all primes $q$ not dividing $|H|$. A finite group $G$ is an $M S$-group if the maximal subgroups of all the Sylow subgroups of $G$ are S-semipermutable in $G$. The aim of the present paper is to characterise the finite MS-groups.

2010 Mathematics subject classification: primary 20D10; secondary 20D15, 20D20.

Keywords and phrases: finite group, soluble PST-group, $\mathrm{T}_{0}$-group, MS-group, BT-group.
\end{abstract}

\section{Introduction}

In the following, $G$ always denotes a finite group. Recall that subgroups $H$ and $K$ of $G$ is said to permute if $H K$ is a subgroup of $G$ and that a subgroup $H$ of $G$ is said to be permutable in $G$ if $H$ permutes with all subgroups of $G$.

Various generalisations of permutability have been defined and studied and, in particular, we mention the S-semipermutability. A subgroup $H$ is said to be $S$-semipermutable in $G$ if $H$ permutes with every Sylow $q$-subgroup of $G$ for all primes $q$ not dividing $|H|$. This subgroup embedding property

*Departament d'Àlgebra, Universitat de València, Dr. Moliner, 50, 46100 Burjassot, València, Spain, email: Adolfo.Ballester@uv.es

${ }^{\dagger}$ Department of Mathematics, University of Kentucky, Lexington KY 40506-0027, USA, email: clark@ms.uky.edu

${ }^{\ddagger}$ Institut Universitari de Matemàtica Pura i Aplicada, Universitat Politècnica de València, Camí de Vera, s/n, 46022 València, Spain, email: resteban@mat.upv.es. Current address: Departament d'Àlgebra, Universitat de València, Dr. Moliner, 50, 46100 Burjassot, València, Spain, email: Ramon.Esteban@uv.es

$\S$ Department of Mathematics, Auburn University at Montgomery, P.O. Box 244023, Montgomery, AL 36124-4023, USA, email: mragland@aum.edu 
has been extensively studied recently (see for instance $[1,4,7,9]$ ). Most of these papers concern situations where many subgroups (for instance all maximal subgroups of the Sylow subgroups) have the stated property. Thus we say that a group $G$ is an MS-group if the maximal subgroups of all the Sylow subgroups of $G$ are S-semipermutable in $G$.

The main aim of this paper is to characterise the MS-groups.

\section{Preliminary results}

In this section, we collect the definitions and results which are needed to prove our main theorems.

We shall adhere to the notation used in [2]: this book will be the main reference for terminology and results on permutability.

A subgroup $H$ is permutable in a group $G$ if and only if $H$ permutes with every $p$-subgroup of $G$ for every prime $p$ (see for instance [2, Theorem 1.2.2]). A less restrictive subgroup embedding property is the S-permutability introduced by Kegel in 1962 [5] and defined in the following way:

Definition 1. A subgroup $H$ of $G$ is said to be $S$-permutable in $G$ if $H$ permutes with every Sylow $p$-subgroup of $G$ for every prime $p$.

Note that we are not considering all $p$-subgroups, but just the maximal ones, that is, the Sylow $p$-subgroups.

In recent years there has been widespread interest in the transitivity of normality, permutability and S-permutability.

Definition 2. 1. A group $G$ is a $T$-group if normality is a transitive relation in $G$, that is, if every subnormal subgroup of $G$ is normal in $G$.

2. A group $G$ is a PT-group if permutability is a transitive relation in $G$, that is, if $H$ is permutable in $K$ and $K$ is permutable in $G$, then $H$ is permutable in $G$.

3. A group $G$ is a PST-group if S-permutability is a transitive relation in $G$, that is, if $H$ is S-permutable in $K$ and $K$ is S-permutable in $G$, then $H$ is S-permutable in $G$.

If $H$ is S-permutable in $G$, it is known that $H$ must be subnormal in $G$ ([2, Theorem 1.2.14(3)]). Therefore, a group $G$ is a PST-group (respectively a PT-group) if and only if every subnormal subgroup is S-permutable (respectively permutable) in $G$. 
Note that T implies PT and PT implies PST. On the other hand, PT does not imply $\mathrm{T}$ (non-Dedekind modular $p$-groups) and PST does not imply PT (non-modular $p$-groups).

A less restrictive class of groups is the class of $T_{0}$-groups which has been studied in $[3,6,8]$.

Definition 3. A group $G$ is called a $T_{0}$-group if the Frattini factor group $G / \Phi(G)$ is a T-group.

The group in Example 10 below is a soluble $\mathrm{T}_{0}$-group which is not a PSTgroup. Soluble $\mathrm{T}_{0}$-groups are closely related to PST-groups as the following result shows.

Theorem 4 ([6, Theorems 5 and 7 and Corollary 3]). Let $G$ be a soluble $T_{0}$-group with nilpotent residual $L=\gamma_{\infty}(G)$. Then:

1. $G$ is supersoluble.

2. $L$ is a nilpotent Hall subgroup of $G$.

3. If $L$ is abelian, then $G$ is a PST-group.

Here the nilpotent residual $\gamma_{\infty}(G)$ of a group $G$ is the smallest normal subgroup $N$ of $G$ such that $G / N$ is nilpotent, that is, the limit of the lower central series of $G$ defined by $\gamma_{1}(G)=G, \gamma_{i+1}(G)=\left[\gamma_{i}(G), G\right]$ for $i \geq 1$.

It is known that S-semipermutability is not transitive. Hence it is natural to consider the following class of groups:

Definition 5. A group $G$ is called a BT-group if S-semipermutability is a transitive relation in $G$, that is, if $H$ is S-semipermutable in $K$ and $K$ is S-semipermutable in $G$, then $H$ is S-semipermutable in $G$.

This class was introduced and characterised by Wang, Li and Wang in [9]. Further contributions were presented in [1].

Theorem 6 ([9, Theorem 3.1]). Let $G$ be a group. The following statements are equivalent:

1. G is a soluble BT-group.

2. Every subgroup of $G$ is $S$-semipermutable.

3. $G$ is a soluble PST-group and if $p$ and $q$ are distinct prime divisors of the order of $G$ not dividing the order of the nilpotent residual of $G$, then $\left[G_{p}, G_{q}\right]=1$, where $G_{p} \in \operatorname{Syl}_{p}(G)$ and $G_{q} \in \operatorname{Syl}_{q}(G)$. 
The group presented in Example 9 below is an MS-group which is not a soluble BT-group. Furthermore, Example 10 shows that the classes of $\mathrm{T}_{0}$-groups and MS-groups are not closed under taking subgroups.

The first remarkable fact concerning the structure of an MS-group can be found in [7]. It is proved there that every MS-group is supersoluble.

Theorem 7 ([7, Corollary 9]). Let $G$ be an MS-group. Then $G$ is supersoluble.

More recently, the second and fourth authors proved the followign theorem.

Theorem 8 ([4, Theorems A, B and C]). Let $G$ be an MS-group with nilpotent residual $L=\gamma_{\infty}(G)$. Then:

1. If $N$ is a normal subgroup of $G$, then $G / N$ is an $M S$-group;

2. $L$ is a nilpotent Hall subgroup of $G$;

3. $G$ is a soluble $T_{0}$-group.

It is well-known that the nilpotent residual of a supersoluble group is nilpotent. Hence the nilpotency of $L$ in Theorem 8 is a consequence of Theorem 7.

Let $G$ be a group whose nilpotent residual $L=\gamma_{\infty}(G)$ is a Hall subgroup of $G$. Let $\pi=\pi(L)$ and let $\theta=\pi^{\prime}$, the complement of $\pi$ in the set of all prime numbers. Let $\theta_{N}$ denote the set of all primes $p$ in $\theta$ such that if $P$ is a Sylow $p$-subgroup of $G$, then $P$ has at least two maximal subgroups. Further, let $\theta_{C}$ denote the set of all primes $q$ in $\theta$ such that if $Q$ is a Sylow $q$-subgroup of $G$, then $Q$ has only one maximal subgroup, or equivalently, $Q$ is cyclic.

Throughout this paper we will use the notation presented above concerning $\pi, \theta=\pi^{\prime}, \theta_{N}$, and $\theta_{C}$.

\section{The main results}

Our first main result is a characterisation theorem.

Theorem A. Let $G$ be a group with nilpotent residual $L=\gamma_{\infty}(G)$. Then $G$ is an MS-group if and only if $G$ satisfies the following properties.

1. $G$ is a $T_{0}$-group.

2. $L$ is a nilpotent Hall subgroup of $G$. 
3. If $p \in \pi$ and $P \in \operatorname{Syl}_{p}(G)$, then a maximal subgroup of $P$ is normal in $G$.

4. Let $p$ and $q$ be distinct primes with $p \in \theta_{N}$ and $q \in \theta$. If $P \in \operatorname{Syl}_{p}(G)$ and $Q \in \operatorname{Syl}_{q}(G)$, then $[P, Q]=1$.

5. Let $p$ and $q$ be distinct primes with $p \in \theta_{C}$ and $q \in \theta$. If $P \in \operatorname{Syl}_{p}(G)$ and $Q \in \operatorname{Syl}_{q}(G)$ and $M$ is the maximal subgroup of $P$, then $Q M=$ $M Q$ is a nilpotent subgroup of $G$.

Proof. Let $G$ be an MS-group. By Theorems 7 and 8, $G$ is a supersoluble $\mathrm{T}_{0}$-group whose nilpotent residual $L$ is a nilpotent Hall subgroup of $G$. Thus properties 1 and 2 hold.

Let $\pi=\pi(L)$ and let $p \in \pi$. Further, let $P$ be a Sylow $p$-subgroup of $G$ and let $M$ be a maximal subgroup of $P$. Then $M \leq P \unlhd L$ and $M$ is normal in $L$ and subnormal in $G$. Let $q \in \theta=\pi^{\prime}$ and note that $M Q$ is a subgroup of $G$ for a given Sylow $q$-subgroup $Q$ of $G$. Moreover $M$ is a Sylow $p$-subgroup of $M Q$ and so $M$ is a normal subgroup of $M Q$. Consequently $M$ normalises $P$ and each Sylow $q$-subgroup $Q$ of $G$, so $M$ is a normal subgroup of $G$ and property 3 holds.

Let $X$ be a Hall $\theta$-subgroup of $G$ and note that $G=L \rtimes X$, the semidirect product of $L$ by $X$, and $X$ is nilpotent. Let $t$ be a prime from $\theta_{N}$ and $r$ be a prime from $\theta$. Also let $T \in \operatorname{Syl}_{t}(G)$ and $R \in \operatorname{Syl}_{r}(G)$. Let $M_{1}$ and $M_{2}$ be two distinct maximal subgroups of $T=\left\langle M_{1}, M_{2}\right\rangle$. Since $G$ is an MS-group, $M_{1} R=R M_{1}$ and $M_{2} R=R M_{2}$. Applying [2, Theorem 1.2.2], we have $R T=T R$. Observe that $T R$ is a $\theta$-subgroup of $G$ and so $T R$ is nilpotent since $T R$ is a subgroup of some conjugate of $X$. Therefore, $[T, R]=1$ and property 4 holds.

Let $p$ and $q$ be distinct primes with $p \in \theta_{C}$ and $q \in \theta$. Further, let $P \in \operatorname{Syl}_{p}(G)$ and $Q \in \operatorname{Syl}_{q}(G)$. If $M$ is the maximal subgroup of $P$, then $Q M=M Q$ is a nilpotent $\theta$-subgroup of $G$. Thus property 5 holds.

Let $G$ be a group satisfying properties $1-5$. We are to show that $G$ is an MS-group. By properties 1 and 2, $G$ is a soluble $\mathrm{T}_{0}$-group, and by Theorem 4 , $G$ is thus supersoluble.

Let $p \in \pi=\pi(L)$, let $P$ be a Sylow $p$-subgroup of $G$, and let $M$ be a maximal subgroup of $P$. Then $M$ is a normal subgroup of $G$ by property 3 and clearly $P$ is a normal subgroup of $G$. This means that $M$ permutes with every Sylow subgroup of $G$ and $P$ permutes with every maximal subgroup of any Sylow subgroup of $G$.

Let $p$ and $q$ be distinct primes from $\theta$ and let $P \in \operatorname{Syl}_{p}(G)$ and $Q \in$ $\operatorname{Syl}_{q}(G)$. We consider a maximal subgroup $M$ of $P$. Note that $\theta=\theta_{N} \cup \theta_{C}$ 
and $\theta_{N} \cap \theta_{C}=\emptyset$, the empty set. If $p \in \theta_{N}$, then by property $4,[P, Q]=1$, so that $M Q=Q M$. Hence assume $p \in \theta_{C}$. Then, by property $5, M Q=Q M$.

Therefore, every maximal subgroup of any Sylow subgroup of $G$ is Ssemipermutable in $G$ and $G$ is an MS-group.

The second and fourth authors in [4] posed the following two questions.

1. When is a soluble PST-group an MS-group?

2. When is a soluble PST-group which is also an MS-group a BT-group?

Using Theorem A we are able to answer the first question and provide a partial answer to the second.

Theorem B. Let $G$ be a soluble PST-group. Then $G$ is an MS-group if and only if $G$ satisfies 4 and 5 of Theorem $A$.

Proof. Let $G$ be a soluble PST-group with nilpotent residual $L=\gamma_{\infty}(G)$. By Lemma 5 of [6], $G / \Phi(G)$ is a T-group and so $G$ is a $T_{0}$-group. Notice that 1, 2 and 3 of Theorem A are satisfied for the group $G$.

Assume that $G$ is an MS-group. By Theorem A, 4 and 5 are satisfied by $G$.

Conversely, assume that 4 and 5 of Theorem A are satisfied by $G$. By Theorem A, $G$ is an MS-group.

This completes the proof.

The group given in Example 9 below is a soluble PST-group which is not an MS-group and the group given in Example 10 is an MS-group which is not a soluble PST-group.

Theorem C. Let $G$ be a soluble PST-group which is also an MS-group. If $\theta_{C}$ is the empty set, then $G$ is a BT-group.

Proof. Let $G$ be a soluble PST-group which is also an MS-group. Let $L=$ $\gamma_{\infty}(G)$ be the nilpotent residual of $G$. By the Theorem of Agrawal [2, Theorem 2.1.8], $L$ is an abelian Hall subgroup of $G$ on which $G$ acts by conjugation as a group of power automorphisms. Recall that $\theta=\pi^{\prime}$, where $\pi=\pi(L)$. Moreover $\theta=\theta_{N}$ if $\theta_{C}$ is empty. Let $p$ and $q$ be distinct primes from $\theta$ and let $P \in \operatorname{Syl}_{p}(G)$ and $Q \in \operatorname{Syl}_{q}(G)$. Note that since $G$ is an MS-group, we have that $G$ satisfies properties 4 and 5 of Theorem A. Then $\left[G_{p}, G_{q}\right]=1$ by property 4 of that theorem. Therefore, $G$ is a BT-group by Theorem 6 . This completes the proof of Theorem C.

We remark that if $\theta$ contains only one prime, then $G$ is a BT-group by Corollary 3.4 of [9]. 


\section{Examples}

The following examples appear in [4]. For the sake of completeness, we list them here.

Example 9. Let $G=\langle y, z, x| y^{3}=z^{2}=x^{7}=1,[y, z]=1, x^{y}=x^{2}, x^{z}=$ $\left.x^{-1}\right\rangle$. Then $\left[\langle y\rangle^{x}, z\right] \neq 1$ and $G$ is a soluble group which is not a BT-group. However, $G$ is an MS-group.

Example 10. Let $G=\langle a, x, y| a^{2}=x^{3}=y^{3}=[x, y]^{3}=[x,[x, y]]=$ $\left.[y,[x, y]]=1, x^{a}=x^{-1}, y^{a}=y^{-1}\right\rangle$, then $H=\langle x, y\rangle$ is an extraspecial group of order 27 and exponent 3. Let $z=[x, y]$, thne $z^{a}=z$. Then $\Phi(G)=$ $\Phi(H)=\langle z\rangle=\mathrm{Z}(G)=\mathrm{Z}(H)$. Note that $G / \Phi(G)$ is a T-group so that $G$ is a $\mathrm{T}_{0}$-group. The maximal subgroups of $H$ are normal in $G$ and it follows that $G$ is an MS-group. Let $K=\langle x, z, a\rangle$. Then $\langle x z\rangle$ is a maximal subgroup of $\langle x, z\rangle$, the Sylow 3-subgroup of $K$. However, $\langle x z\rangle$ does not permute with $\langle a\rangle$ and hence $\langle x z\rangle$ is not an S-semipermutable subgroup of $K$. Therefore, $K$ is not an MS-subgroup of $G$. Also note that $\Phi(K)=1$ and so $K$ is not a T-subgroup of $G$ and $K$ is not a $\mathrm{T}_{0}$-subgroup of $G$. Hence the class of soluble $\mathrm{T}_{0}$-groups is not closed under taking subgroups. Note that $G$ is a soluble group which is not a PST-group.

Example 11. Let $G=\langle y, z, x| y^{9}=z^{2}=x^{19^{2}}=1,[y, z]=1, x^{y}=x^{62}, x^{z}=$ $\left.x^{-1}\right\rangle$. Then the soluble group $G$ is a PST-group, but $G$ is not an MS-group since $\left[\left\langle y^{2}\right\rangle^{x}, z\right] \neq 1$.

\section{Acknowledgements}

The work of the first and the third authors has been supported by the grant MTM2010-19938-C03-03 from the Ministerio de Economía y Competitividad, Spain. The first author has also been supported by the grant 11271085 from the National Natural Science Foundation of China.

\section{References}

[1] K. A. Al-Sharo, J. C. Beidleman, H. Heineken, and M. F. Ragland. Some characterizations of finite groups in which semipermutability is a transitive relation. Forum Math., 22(5):855-862, 2010. Corrigendum in Forum Math., 24(6):1333-1334, 2012. 
[2] A. Ballester-Bolinches, R. Esteban-Romero, and M. Asaad. Products of finite groups, volume 53 of de Gruyter Expositions in Mathematics. Walter de Gruyter, Berlin, 2010.

[3] A. Ballester-Bolinches, R. Esteban-Romero, and M. C. Pedraza-Aguilera. On a class of p-soluble groups. Algebra Colloq., 12(2):263-267, 2005.

[4] J. C. Beidleman and M. F. Ragland. Groups with maximal subgroups of Sylow subgroups satisfying certain permutability conditions. Southeast Asian Bull. Math., in press.

[5] O. H. Kegel. Sylow-Gruppen und Subnormalteiler endlicher Gruppen. Math. Z., 78:205-221, 1962.

[6] M. F. Ragland. Generalizations of groups in which normality is transitive. Comm. Algebra, 35(10):3242-3252, 2007.

[7] Y. C. Ren. Notes on $\pi$-quasi-normal subgroups in finite groups. Proc. Amer. Math. Soc., 117:631-636, 1993.

[8] R. W. van der Waall and A. Fransman. On products of groups for which normality is a transitive relation on their Frattini factor groups. Quaestiones Math., 19:59-82, 1996.

[9] L. Wang, Y. Li, and Y. Wang. Finite groups in which (S-)semipermutability is a transitive relation. Int. J. Algebra, 2(1-4):143152, 2008. Corrigendum in Int. J. Algebra, 6(13-16):727-728, 2012. 